\title{
Steroid Use is Not Associated with Infection or Wound Complications in Periarticular Fractures
}

Christopher A Staley, Mara L. Schenker, Wesley A. Glick, Alexandra Ernst, Victoria Kravets, Brent Wise, Thomas Moore Jr. Emory University, Grady Memorial Hospital

\section{INTRODUCTION}

- The aim of this study was to determine the impact of systemic steroid use on rates of infection, wound healing, reoperation, and readmission in patients with periarticular orthopedic trauma

\section{METHODS}

- A retrospective study was conducted utilizing the NSQIP database

- 26,174 patients aged 18-89 who sustained a periarticular fracture of the ankle, knee or elbow from 2006-2015 were included

- Those presenting with preadmission systemic sepsis and disseminated cancer were excluded

- Those in the steroid group (534 patients) are defined as patients who require the regular administration of oral or parenteral corticosteroid medications or immunosuppressant medications, within 30 days prior to the principle operative procedure for a chronic medical condition

- A subset of patients $(n=6,151)$ with the most common injuries (distal femur, tibial plateau, tibial pilon, and distal humerus fractures) was also analyzed separately

- Chi square, univariate, and multivariate logistic regression were performed to determine the association between steroid use for a chronic condition and infection, reoperation, and readmission

\section{RESULTS}

\begin{tabular}{|c|c|c|}
\hline \multirow{2}{*}{$\overline{\operatorname{Sex}}$} & Female & $15661(59.9 \%)$ \\
\hline & Male & $10500(40.1 \%)$ \\
\hline \multirow{15}{*}{ Ethnicity } & American Indian or Alaska Native & $29(0.1 \%)$ \\
\hline & American Indian or Alaska & $207(0.8 \%)$ \\
\hline & Asian & $599(2.3 \%)$ \\
\hline & Asian or Pacific Islander & $6(0 \%)$ \\
\hline & Black or African American & $2309(8.8 \%)$ \\
\hline & Black, Not of Hispanic Origin & $31(0.1 \%)$ \\
\hline & Hispanic, Color Unknown & $29(0.1 \%)$ \\
\hline & Hispanic, White & $49(0.2 \%)$ \\
\hline & Native Hawaiian or Pacifi & $104(0.4 \%)$ \\
\hline & Native Hawaiian or Pacific Islander & $15(0.1 \%)$ \\
\hline & NULL & $47(0.2 \%)$ \\
\hline & Unknown & $856(3.3 \%)$ \\
\hline & Unknown/Not Reported & 4366 (16.7\%) \\
\hline & White & $17310(66.1 \%)$ \\
\hline & White, Not of Hispanic Origin & $217(0.8 \%)$ \\
\hline \multirow{8}{*}{ Age } & $<21$ & $810(3.1 \%)$ \\
\hline & $21-30$ & 3315 (12.7\%) \\
\hline & $31-40$ & 3309 (12.6\%) \\
\hline & $41-50$ & 3844 (14.7\%) \\
\hline & $51-60$ & $5157(19.8 \%)$ \\
\hline & $61-70$ & $4663(17.8 \%)$ \\
\hline & $71-80$ & 2910 (10.9\%) \\
\hline & $81-90$ & $1636(6.2 \%)$ \\
\hline
\end{tabular}

\section{RESULTS}

Table 2. All Patients Analysis

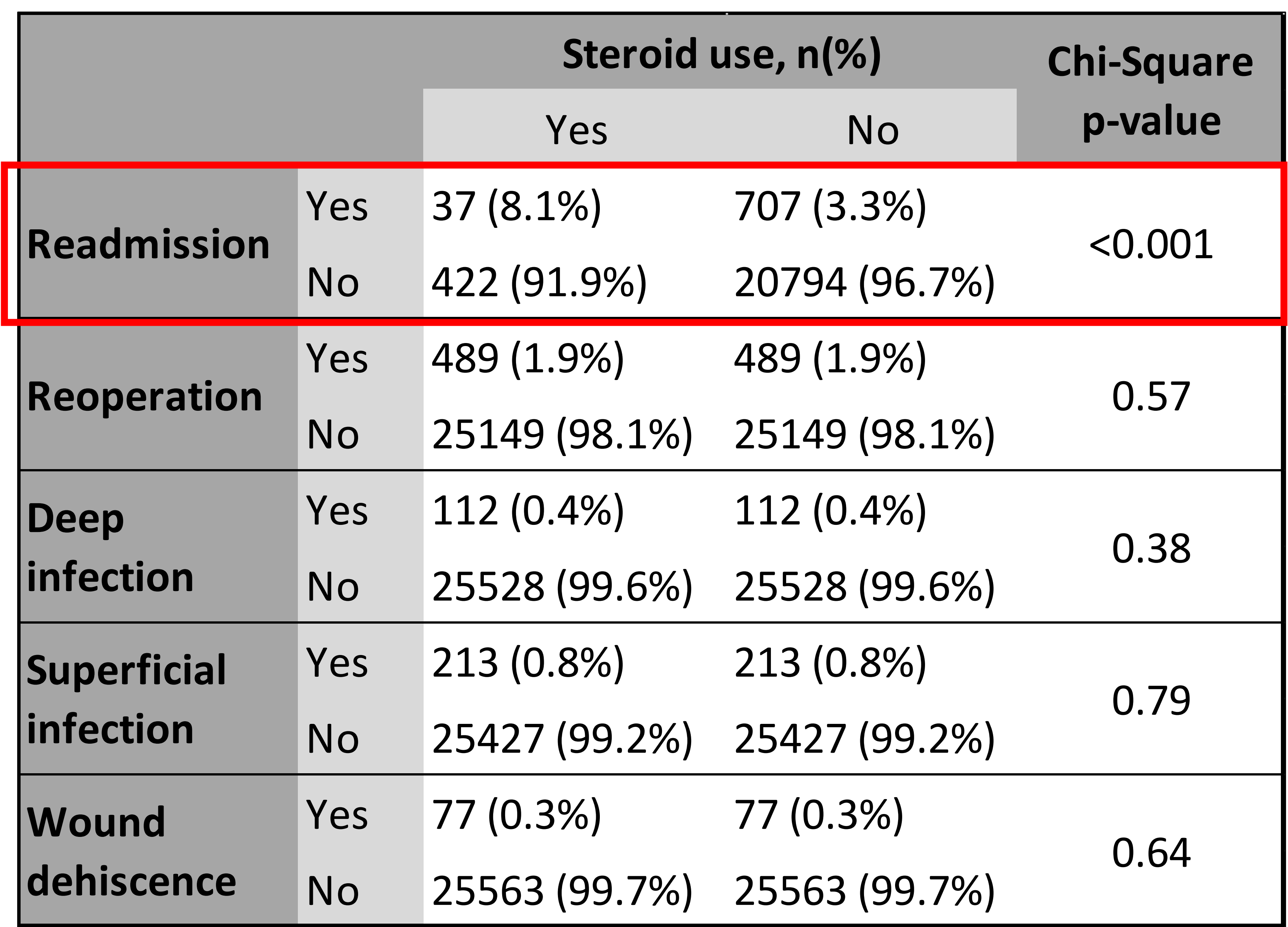

Table 3. Most Common Injury Analysis

\begin{tabular}{|c|c|c|c|c|}
\hline & & \multicolumn{2}{|c|}{ Steroid use, n(\%) } & \multirow{2}{*}{$\begin{array}{c}\text { Chi-square } \\
\text { p-value }\end{array}$} \\
\hline & & Yes & No & \\
\hline \multirow{2}{*}{$\begin{array}{l}\text { Deep } \\
\text { infection }\end{array}$} & Yes & $0(0 \%)$ & $43(0.7 \%)$ & \multirow{2}{*}{0.24} \\
\hline & No & 189 (100\%) & 5919 (99.3\%) & \\
\hline \multirow{2}{*}{$\begin{array}{l}\text { Superficial } \\
\text { infection }\end{array}$} & Yes & $0(0 \%)$ & $65(1.1 \%)$ & \multirow{2}{*}{0.15} \\
\hline & No & 189 (100\%) & 5897 ( 98.9\%) & \\
\hline \multirow{2}{*}{$\begin{array}{l}\text { Wound } \\
\text { dehiscence }\end{array}$} & Yes & $0(0 \%)$ & $21(0.4 \%)$ & \multirow{2}{*}{0.41} \\
\hline & No & 189 (100\%) & 5941 (99.6\%) & \\
\hline
\end{tabular}

- After controlling for co-variates, deep infection $(p=0.26)$, superficial infection $(p=0.74)$, wound dehiscence $(p=0.40)$, and reoperation $(p=0.51)$ remained insignificant on multivariate regression between groups, while those requiring chronic steroid administration had increased risk of 30-day readmission (OR 1.64, 95\% CI 1.15-2.34, $\mathrm{p}=0.006$ ).

\section{CONCLUSION}

- Administration of systemic corticosteroids in the orthopedic patient has been proposed as a means of improving analgesia via a multimodal approach, and potentially accelerating definitive surgical timing by reducing soft tissue swelling.

- This study demonstrates that in traumatic periarticular fractures, those who receive corticosteroids for a chronic condition are not at an increased risk of developing infection, wound dehiscence, or reoperation.

- This provides sufficient equipoise to help direct future prospective studies to evaluate potential steroid benefits. 